\title{
Geophysical Research Letters
}

\section{RESEARCH LETTER \\ 10.1002/2014GL060313 \\ Key Points: \\ - New measurements and a model reveal unmeasured physics of katabatic flow \\ - Outer layer pressure effects are significant in low-speed highly variable wind \\ - Explicit treatment of the canopy is important in thin steep-slope katabatic jets}

Supporting Information:

- Readme

- Figure S1

- Figure S2

- Figure S3

- Table S1

- Table S2

- Table S3

- Table S4

- Text S1

- Text S2

- Text S3

Correspondence to: H. J. Oldroyd,

holly.oldroyd@epfl.ch

\section{Citation:}

Oldroyd, H. J., G. Katul, E. R. Pardyjak, and M. B. Parlange (2014), Momentum balance of katabatic flow on steep slopes covered with short vegetation, Geophys. Res. Lett., 41, 4761-4768, doi:10.1002/2014GL060313.

Received 23 APR 2014 Accepted 13 JUN 2014 Accepted article online 18 JUN 2014 Published online 9 JUL 2014

\section{Momentum balance of katabatic flow on steep slopes covered with short vegetation}

\author{
Holly J. Oldroyd ${ }^{1}$, Gabriel Katul' ${ }^{2}$ Eric R. Pardyjak ${ }^{3}$, and Marc B. Parlange ${ }^{1,4}$ \\ ${ }^{1}$ School of Architecture, Civil and Environmental Engineering, École Polytechnique Fédérale de Lausanne, Lausanne, \\ Switzerland, ${ }^{2}$ Nicholas School of the Environment, Duke University, Durham, North Carolina, USA, ${ }^{3}$ Department of \\ Mechanical Engineering, University of Utah, Salt Lake City, Utah, USA, ${ }^{4}$ Department of Civil Engineering, University of \\ British Columbia, Vancouver, British Columbia, Canada
}

\begin{abstract}
Katabatic flows over alpine mountainous terrain differ from their forested or bare slope counterparts due to the presence of well-ventilated, short vegetation. The impact of a grass canopy and larger-scale pressure perturbations on the one-dimensional mean momentum balance is explored via theory and field measurements. The model presented here reproduces the measured velocity jet shape and turbulent flux gradients. These two features imply that even when Monin-Obuhkov similarity theory breaks down, its use for a stability adjusted mixing length remains effective to first order. Results reveal that outer layer pressure effects can be significant under low-speed wind conditions at the top of the thin katabatic layer when larger variations in the wind direction are observed. An analytical expression to estimate the jet height, which can be utilized in large-scale weather prediction models, shows the importance of including canopy effects for the thin katabatic flow region above the vegetation.
\end{abstract}

\section{Introduction}

Katabatic winds in mountainous terrain are drawing increased attention given their role in transporting heat [Monti et al., 2002], water vapor, pollutants [Pardyjak et al., 2009], and $\mathrm{CO}_{2}$ (especially, if vegetated or near urban environments) [Sun et al., 2007] and in the formation of frost [Laughlin and Kalma, 1987], fog [Duynkerke, 1999], and cold pools [Whiteman et al., 2001] within basins and valleys. Typically, katabatic winds form over sloping terrain during nights characterized by clear-sky conditions and weak synoptic forcing. Radiative cooling at the surface generates stable stratification and negative buoyancy thereby causing cooler air to sink down the inclined surface [Whiteman, 2000]. Nocturnal drainage flow within and above tall (forest) canopies on slopes has received significant attention [e.g., Chen and Yi, 2012; Luo et al., 2009; Luo and Li, 2009], especially in the context of ecosystem carbon balance closure [e.g., Burns et al., 2011; Turnipseed et al., 2003]. Additionally, slopes with forested canopies have been shown to produce complicated mean flow structures, having jet peaks above or below the canopy top [Burns et al., 2011; Yi et al., 2005; Froelich et al., 2005; Froelich and Schmid, 2006]). However, the effects of short vegetation over steep slopes have been largely ignored or lumped into a roughness length that is typically an order of magnitude smaller than the canopy height itself. It has been known for some time that katabatic flows over steep slopes pose unique challenges to their inclusion within large-scale numerical models. First, models of the stable atmospheric boundary layer often "break down" due to a range of physical phenomena that are not commonly included in turbulence modeling, from the formation of a low-level jet to larger-scale motions that propagate to the surface [Mahrt et al., 2001; Mahrt, 1998]. In addition, numerical models of the stable boundary layer are prone to producing nonphysical phenomena such as "run-away" surface cooling and spurious laminarization [Jiménez and Cuxart, 2005; Mahrt, 1998]. Second, numerical models generally implement similarity-based scaling parameterizations, usually adopting Monin-Obukhov similarity theory to link the surface to the flow. These parameterizations, developed for flat terrain [e.g., Pahlow et al., 2001; Brustaert, 1982] with a constant flux surface layer, are known to break down in the presence of katabatic flows [Nadeau et al., 2012]. Third, steep, alpine mountainous slope flows typically result in near-surface jets with a peak jet velocity close to the ground surface, thereby compounding the common problems of vertical grid resolution and the explicit coupling between the land surface and the flow aloft in a coarse grid [Renfrew, 2004]. 


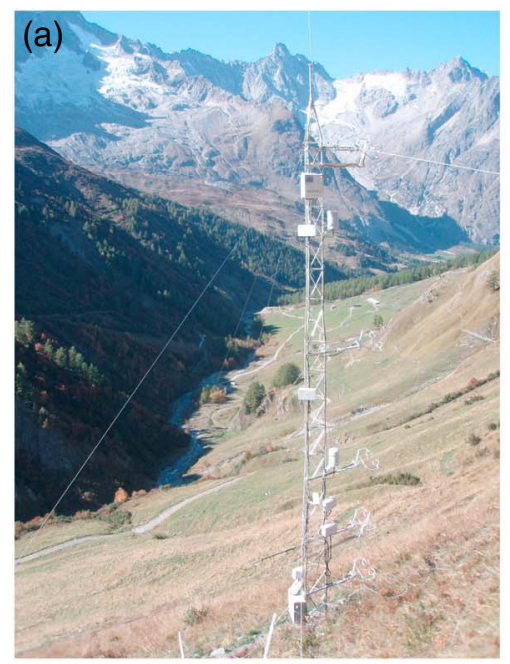

(b)

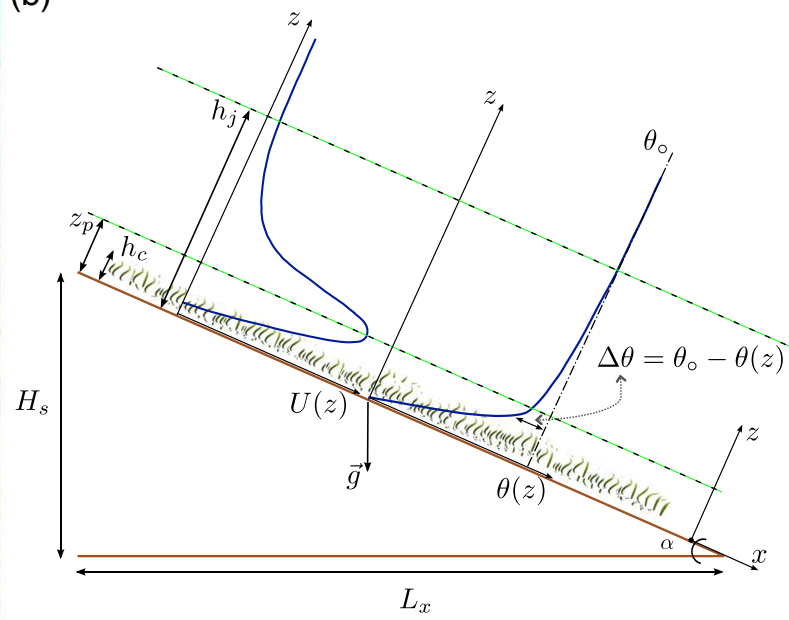

Figure 1. (a) Alpine slope experiment site showing the flux tower. (b) Schematic of slope-aligned coordinate system including sketches of a theoretical katabatic jet and virtual temperature profile (see text for label descriptions).

Existing layer models of katabatic wind vary in complexity and structural description, from bulk parameterizations [e.g., Mahrt, 1982; Manins and Sawford, 1979] to those that describe mean (velocity and temperature) [e.g., Garrett, 1983; Yamada, 1983] and turbulent flux (momentum and heat) profiles [e.g., Rao and Snodgrass, 1981; Yi et al., 2005]. However, only a handful of models have been compared with measurements over steep slopes $\left(>15^{\circ}\right)$. Furthermore, since few measurements have been made of the detailed turbulent structure within the katabatic layer, especially over steep terrain [Nadeau et al., 2013], models are rarely compared with turbulent flux measurements [Yi et al., 2005]. These comparisons are necessary because even if the katabatic layer is shallow (as over steep slopes), mass, momentum, and heat exchanges between the atmosphere and canopy-ground system occur within this layer [Mahrt et al., 2001].

Here a one-dimensional mean momentum balance formulation is developed that is forced with measured air temperature profiles. This model is used in conjunction with multilevel measurements over a steep, grassy, alpine slope to interpret the mean momentum balance and characterize the katabatic jet. A key feature of this model is that it explicitly includes the role of short vegetation (as previously considered by Garrett [1983], Luo et al. [2009], and Luo and Li[ 2009], for a forested canopy), and it is shown that even for short canopies, the role of canopy height remains significant. In addition, the model includes a simplified pressure parameterization that accounts for weak larger-scale (synoptic, mesoscale, and/or valley scale) pressure perturbations originating from the outer layer and penetrating to the surface. This type of pressure penetration has been studied in flows over hills [Belcher and Wood, 1996; Raupach and Finnigan, 1997] and can also account for some of the variation of topographic curvature over extensive slopes [Haiden and Whiteman, 2005]. Using measurements from nine different intensive observation nights, the significance of including such outer pressure parameterization on the form and structure of the katabatic flow is explored.

\section{Field Experiment}

During the summer of 2011 in Val Ferret [Simoni et al., 2011], a narrow alpine valley in Switzerland, instruments were deployed to investigate thermally driven steep slope flows as a follow-up experiment [see Nadeau et al., 2013]. The local downslope angle, determined from a $10 \mathrm{~m}^{2}$ area of a $1 \mathrm{~m}$ resolution digital elevation map, is $35.5^{\circ}$. Vegetation along the slope was $\sim 30 \mathrm{~cm}$ tall alpine flowers and grasses. Measurements were sampled at $20 \mathrm{~Hz}$ from five triaxial sonic anemometers (CSAT3, Campbell Scientific) that were mounted with a slope-normal tilt from vertical (to reduce tilt correction angles and verified with an inclinometer) on a $10 \mathrm{~m}$ tower at slope-normal heights of $0.45,1.27,2.15,3.79$, and $6.32 \mathrm{~m}$ (see Figure 1a). Velocity tilt corrections were made with the planar fit method applied to each of six $40^{\circ}$ wind sectors [Wilczak et al., 2001; Foken, 2008] (see supporting information for details regarding the sensor tilt corrections). Additionally, a thermocouple array measuring 1 min mean air temperatures was mounted at the tower site. These measurements were converted to virtual potential temperatures, $\theta$, and used to drive the thermal forcing in the model described herein (see supporting information for more details about the 
temperature measurements). Figure $1 \mathrm{~b}$ introduces the slope-aligned coordinate system and provides a schematic for the shallow katabatic jet and the near-surface temperature profile that drives the flow. The schematic includes characteristic heights such as the canopy height, $h_{c}$, the jet layer height, $h_{j}$, and the jet peak height, $z_{p}$, and the virtual temperature deficit, $\Delta \theta$. Measurements from nine clear-sky nights in September of 2011 when downslope flow was observed were used in conjunction with the model. Note that the flow exhibits no consistent jet shape in the $U(z)$ profile during the nights of 24,29 , and 30 September, indicating a significant larger-scale disturbance preventing a katabatic jet formation. Table S4 in the supporting information summarizes characteristic quantities for the experiments. A cospectral analysis (not included herein) shows that due to sensor path length and sampling frequency limitations, the measured turbulent fluxes at the lowest measurement location are attenuated and their magnitudes are underestimated. This underestimation should not be overlooked in data-model comparisons.

\section{Theory}

\subsection{Definitions and Governing Equations}

For a constant slope with no curvature and ignoring Coriolis effects near the ground surface, the mean longitudinal and surface-normal momentum balance equations for a katabatic flow on a slope uniformly covered with vegetation (adapted from Haiden and Whiteman [2005] to include the canopy drag) are

$$
\frac{\partial U}{\partial t}+U \frac{\partial U}{\partial x}+V \frac{\partial U}{\partial y}+W \frac{\partial U}{\partial z}=-\frac{1}{\rho_{o}} \frac{\partial P}{\partial x}+g \frac{\Delta \theta}{\theta_{o}} \sin (\alpha)-\frac{\partial \overline{u^{\prime} W^{\prime}}}{\partial z}-C_{d} a(z)|U| U
$$

and

$$
\frac{\partial W}{\partial t}+U \frac{\partial W}{\partial x}+V \frac{\partial W}{\partial y}+W \frac{\partial W}{\partial z}=-\frac{1}{\rho_{o}} \frac{\partial P}{\partial z}-g \frac{\Delta \theta}{\theta_{o}} \cos (\alpha)-C_{d} a(z)|U| W
$$

where $t$ is time, $x, y$, and $z$ are aligned directions so that $x$ is the streamwise direction; $y$ is the spanwise direction, and $z$ is orthogonal to the ground; $U, V$, and $W$ are the mean velocity components along $x, y$, and $z$, respectively; $\alpha$ is the slope angle; $H_{s}$ and $L_{x}$ are characteristic height and length of the slope, respectively, $\left(\tan (\alpha)=H_{s} / L_{x}\right) ; P$ is the mean pressure perturbation; $g$ is the gravitational acceleration; $\rho_{o}$ and $\theta_{o}$ are the background density and air temperature above the katabatic flow region (hereafter, quantities subscripted with $o$ indicate averaged variables outside the katabatic jet); $\Delta \theta$ is the virtual temperature deficit (difference from the background state) within the katabatic flow region; $u^{\prime}$ and $w^{\prime}$ are turbulent excursions from the mean state; an overbar represents time averaging; $C_{d}$ is the drag coefficient of the foliage defining the canopy; and $a(z)$ is the leaf area density of the vegetation assumed to be uniformly covering the slope and is represented here as $\mathrm{LAl} / h_{c}$, where $\mathrm{LAl}$ is the leaf area index and $h_{c}$ is the mean vegetation height. The terms $\partial \overline{u^{\prime} u^{\prime}} / \partial x, \partial \overline{u^{\prime} v^{\prime}} / \partial y$, and $\partial \overline{w^{\prime} w^{\prime}} / \partial z$ are ignored relative to other terms in the respective mean momentum balance equations given that the turbulence is generally weak under such conditions. Note, however, that the $\partial \overline{w^{\prime} w^{\prime}} / \partial z$ term may play a role not considered here. The model coordinate system is aligned so that $V=0$ and $W=0$ (for consistency with the field experiment, see supporting information for further justification); lateral homogeneity is also assumed locally along with stationarity so that

and

$$
0=-\frac{1}{\rho_{o}} \frac{\partial P}{\partial x}+g \frac{\Delta \theta}{\theta_{o}} \sin (\alpha)-\frac{\partial \overline{u^{\prime} w^{\prime}}}{\partial z}-C_{d} a(z)|U| U
$$

$$
0=-\frac{1}{\rho_{o}} \frac{\partial P}{\partial z}-g \frac{\Delta \theta}{\theta_{o}} \cos (\alpha)
$$

Upon integrating the momentum balance for $W$ along the $z$ direction from the ground, $z=0$, up to the layer encompassing the katabatic flow region, $z=h_{j}$,

$$
\frac{P\left(h_{j}, x\right)}{\rho_{o}}=-\frac{g}{\theta_{o}} \cos (\alpha) \int_{0}^{h_{j}} \Delta \theta(z) \mathrm{d} z+\frac{P(0, x)}{\rho_{o}} .
$$

Here the term $P(0, x)$ is an integration constant that reflects possible surface pressure variations along the $x$ direction originating from a number of factors such as larger-scale disturbances. The $h_{j}$ is commonly delineated by the height at which $\Delta \theta(z)=0$, and this delineation is employed here. Substituting this estimate of $P(x)$ into equation (3) yields:

$$
0=\frac{g}{\theta_{o}}\left(\sin (\alpha) \Delta \theta(z)+\cos (\alpha) \frac{\partial \int_{0}^{h_{j}} \Delta \theta(z) d z}{\partial x}\right)-\frac{\partial \overline{u^{\prime} w^{\prime}}}{\partial z}-C_{d} a(z)|U| U-\frac{1}{\rho_{o}} \frac{\partial P(0, x)}{\partial x}
$$


The interpretation of the terms are as follows: the first is the hydrostatic pressure driving the katabatic flow downslope, the second is known as the thermal wind arising from the $W$ mean momentum balance, the third is the Reynolds stress gradient, the fourth is the canopy drag force, finite only for $z<h_{c}$, and the last term reflects pressure undulations originating from larger-scale disturbances in the outer layer (i.e., $z / h_{j}>>1$ ) impacting the katabatic flow regime locally.

\subsection{Closure Models}

Even when $\Delta \theta(z)$ is known, equation (6) remains unclosed necessitating another relation between $U$ and $\overline{u^{\prime} w^{\prime}}$. Here a first-order closure is adopted and is given as $\overline{u^{\prime} w^{\prime}}=-K_{t} \partial U / \partial z$, where $K_{t}=I_{m}^{2}|\partial U / \partial z|$ and $I_{m}$ is an effective mixing length specified as $\kappa(z-d) / \phi_{m}(\xi)$ for $z / h_{c}>1$ and $\kappa\left(h_{c}-d\right) / \phi_{m}\left(\xi_{c}\right)$ for $z / h_{c}<1$, where $\phi_{m}(\xi)=1+5 \xi, \xi=(z-d) / L_{m o}$ and $\xi_{c}=\left(h_{c}-d\right) / L_{m o}, L_{m o}$ is the measured Obukhov length defined above the jet region, $\kappa=0.4$ is the von Kármán constant, and $d$ is the zero-plane displacement height of the vegetation determined from the centroid of the drag force acting on the flow and is given as

$$
d=\frac{\int_{0}^{h_{c}} z C_{d} a U(z)^{2} \mathrm{~d} z}{\int_{0}^{h_{c}} C_{d} a U(z)^{2} \mathrm{~d} z}
$$

With these closure assumptions, and upon specifying $\Delta \theta(z)$ (from measurements), the mean longitudinal momentum balance can be solved for $\overline{u^{\prime} w^{\prime}}$ and $U$ provided $\partial P(0, x) / \partial x$ is known (or ignored) and boundary conditions are specified. The boundary conditions imposed are $U(0)=0$ (no-slip at the ground), and $U\left(z_{\max }\right)=U_{o}=U_{5}$, where $z_{5}>h_{j}$ is a reference height and $U_{o}$ is taken as a reference velocity (specified from measurements, $U_{5}$ ) above the jet reflecting outer layer conditions. Additionally, the canopy is assumed to be characterized by a dimensionless foliage drag, $C_{d}=0.2$, and typical LAI $=1.5 \mathrm{~m}^{2} / \mathrm{m}^{2}$ [Katul et al., 2004].

\subsection{Scaling Analysis}

Even for the idealized setup considered here, difficulties in analyzing drainage flows on steep slopes covered with vegetation remain. These difficulties originate from a multiplicity of length scales impacting the mean momentum balance. The pertinent length scales include $L_{x}=H_{s}(\tan (\alpha))^{-1}$ that typically governs horizontal gradients, the thickness of the katabatic flow region, $h_{j}$, that impacts vertical gradients above the canopy but within the jet, the adjustment length scale, $L_{c}=h_{c}\left(C_{d} \mathrm{LAl}\right)^{-1}$, the canopy height, $h_{c}$, affecting the flow within the canopy, and the Obukhov length, $L_{m o}$, dictating the eddy sizes responsible for vertical mixing of momentum. The relative importance of the thermal wind to the hydrostatic pressure term is given as

$$
\frac{\cos (\alpha)}{\sin (\alpha)} \frac{\frac{\partial \int_{0}^{h_{j}} \Delta \theta(z) \mathrm{d} z}{\partial x}}{\Delta \theta(z)} \sim \frac{\cos (\alpha)}{\sin (\alpha)} \frac{h_{j}}{L_{x}} .
$$

For the steep slopes here, $h_{j}$ is of order $10 \mathrm{~m}$ (as shown later), while $L_{x}$ is of order $1000 \mathrm{~m}$. Hence, the thermal wind term may be ignored relative to the hydrostatic term. Moreover, the local distortion time scale of the mean flow by advective terms are on the order of $L_{x} / U$ while vertical gradients of the mean flow within the katabatic region equilibrate with the stresses on time scales of $h_{j} / u_{*}$, where $u_{*}$ is the friction velocity above the canopy but within the katabatic flow region. Hence, provided $\left(h_{j} / L_{x}\right)\left(U / u_{*}\right)<<1$, the advective terms impact the mean velocity profile at time scales much longer than the shear gradients, and the two terms are prohibited from interacting locally. In general, $U / u_{*}$ can be of order 10, but $h_{j} / L_{x}$ is of order $1 \times 10^{-3}$. In the event that $\left(h_{j} / L_{x}\right)\left(U / u_{*}\right)$ is of order unity, advective terms cannot be ignored and the problem can no longer be treated as one-dimensional. It has been known for sometime that a drainage flow immediately above the surface is influenced by the local slope on the smallest scales, while the flow at the higher levels in the atmosphere may be influenced by terrain slopes on a larger scale. Larger-scale disturbances may originate from variations in outer layer mean wind direction, thereby sensing slope angles different from $\alpha$ during the course of an averaging period. These large-scale variations in slope angles are occurring over distances much larger than $h_{j}$ and impact the outer layer flow field far above the katabatic flow region. Under those conditions, and as is common in studies of flow over hills, a scaling analysis for the outer layer well above the katabatic flow region suggests that

$$
-\frac{1}{\rho_{o}} \frac{\partial P(0, x)}{\partial x}=U_{o} \frac{\partial U_{o}}{\partial x}+\frac{\partial U_{o}}{\partial t} \sim C_{p} \frac{U_{o}^{2}}{R},
$$

where $C_{p}$ is a pressure coefficient, $U_{o}$ is, as before, the typical outer layer velocity [Belcher and Wood, 1996], and $R$ is an effective curvature (positive or negative) reflecting slope changes along $L_{x}$ sensed by the 
larger-scale wind [Haiden and Whiteman, 2005]. For a uniform slope, $R \rightarrow \infty$, and the usual assumptions invoked in earlier studies are recovered. With these simplifications,

$$
0=\frac{g}{\theta_{o}} \sin (\alpha) \Delta \theta(z) \mp C_{p} \frac{U_{o}^{2}}{R}+\frac{\partial}{\partial z}\left(I_{m}^{2}\left|\frac{\partial U}{\partial z}\right| \frac{\partial U}{\partial z}\right)-C_{d} a(z)|U| U .
$$

Hence, larger-scale variations in wind direction lead to different slope angles being sensed in the outer layer well above $h_{j}$ that then produces a $\partial P(x) / \partial x$ that integrates these variations at a point. To first order, it may be assumed that $R \sim L_{x}$. In the data analysis here, conditions where the stability regime near the top of $h_{j}$ is strong (and hence susceptible to such larger-scale disturbances) or weak (and bulk flow is near neutral above the jet with a preset direction) are both explored. It is common practice to replace the canopy vertical structure with a momentum roughness height $\left(z_{o}\right)$ while ensuring that $\overline{u^{\prime} w^{\prime}}\left(z_{o}\right)=\overline{u^{\prime} w^{\prime}}\left(h_{c}\right)$. Here $h_{c}$ was retained (via the drag force) thereby allowing the role of $h_{c}$ in modifying aspects of the jet shape such as the location of the maximum mean velocity $\left(z=z_{p}\right)$ to be assessed. To illustrate, consider a linear temperature profile given as $\Delta \theta(z)=\Delta \theta_{\max }\left(1-z / h_{j}\right)$ when $h_{c} / h_{j}<1$ and $\Delta \theta(z)=0$ when $z>h_{j}$. For $h_{c}<z<h_{j}$, where the location of the maximum jet velocity is anticipated, equation (10) can be integrated to yield

$$
\overline{u^{\prime} w^{\prime}}(z)=\overline{u^{\prime} w^{\prime}}\left(h_{c}\right)+\left(A_{D} \mp B_{P}\right)\left(z-h_{c}\right)+A_{D} \frac{h_{c}^{2}-z^{2}}{2 h_{j}},
$$

where $A_{D}=\left(g / \theta_{o}\right) \sin (\alpha) \Delta \theta_{\max }$ and $B_{P}=C_{p} U_{o}^{2} / R$ are constants independent of $z$. The maximum jet velocity $U_{\max }$ occurs at a $z=z_{p}$ where $\partial U / \partial z=0$. From the closure equation $\left.\overline{\left(u^{\prime} w^{\prime}\right.}=-K_{t} \partial U / \partial z\right)$, it follows that $z_{p}$ is colocated with the height at which $\overline{u^{\prime} w^{\prime}}(z)=0$. Using this condition and solving for $z_{p}$ from equation (11) results in

$$
\frac{z_{p}}{h_{j}}=\left[1 \mp \frac{B_{p}}{A_{D}}-\sqrt{\left(-\frac{h_{c}}{h_{j}}+1 \mp \frac{B_{p}}{A_{D}}\right)^{2}+2 \frac{\overline{u^{\prime} w^{\prime}}\left(h_{c}\right)}{A_{D} h_{j}}}\right],
$$

which now makes explicit the role of $h_{c} / h_{j}$. A finite $h_{c}$ displaces the vertical location of the jet peak velocity downward compared to the case when $h_{c}=0$. Likewise, when $B_{p}$ is negative and increasing in magnitude, $z_{p}$ decreases. Also, for negligible $B_{p}$ and small $A_{D}$ associated with a small $\Delta \theta_{\max }, z_{p}$ increases with declining $\Delta \theta_{\max }$. For illustration, consider the case with $B_{p}=0, h_{j}=6 \mathrm{~m}, h_{c}=0.3 \mathrm{~m}, \Delta \theta_{\max } / \theta_{o}=0.01, \alpha=35.5^{\circ}$, and $\overline{u^{\prime} w^{\prime}}\left(h_{c}\right)=-0.05 \mathrm{~m}^{2} \mathrm{~s}^{-2}$. Using equation (12), $z_{p} / h_{j} \approx 0.2$ resulting in $z_{p} / h_{c} \approx 4$ (i.e., of order unity). It is for this reason that the vertical structure of the canopy cannot be entirely ignored for katabatic flows on steep slopes covered even by short vegetation.

\section{Results and Discussion}

Figure 2 shows the comparison between modeled and measured mean velocity and vertical momentum flux profiles for varying pressure coefficient $\left(C_{p}=0,5,10\right)$, along with the measured mean air temperature profile used to force the buoyancy term for the night of 15 September. The model with optimal $C_{p}$ captures the shape of the katabatic jet and its peak velocity near the vegetation top. In addition, it reproduces the large gradients observed in the vertical momentum flux and the sign change that occurs in the vicinity of the jet peak. The largest disagreement between the model and measurements for $\overline{u^{\prime} w^{\prime}}$ occurs at the lowest measurement location, where measured flux attenuation determined from cospectral analysis was also observed. This underestimation in the measured flux magnitudes explains some of the difference, but bias in the modeled results could also exist due to uncertainties in the canopy height or temperature measurements. However, the general agreement infers that even in the absence of a constant flux surface layer, the use of Monin-Obukhov similarity theory to set the stability adjusted mixing length in a K-theory closure model can produce reasonable (first-order) estimations for $\overline{u^{\prime} w^{\prime}}$. Assuming sufficient vertical grid resolution to resolve the extremely thin jet layer, the use of K-theory may be valid for vertical momentum transport if the advection time scale is much longer than the equilibration time scale of turbulence. From a scaling analysis perspective, the advection time $\sim L_{x} / U_{o}$ and the equilibration time with the mean velocity gradient is on the order of the turbulent kinetic energy normalized by its dissipation rate $\left(\sim I_{m} / \sigma_{w}\right)$. Hence, when $L_{x} / I_{m}>U_{o} / \sigma_{w}$, K-theory closure may be used. For the setup here, $L_{x} / I_{m} \sim 100$, while $U_{o} / \sigma_{w} \sim 10$ perhaps suggesting the use of K-theory to close one of the terms in the mean momentum balance is justifiable. The momentum flux profile within the canopy region differs from the expected monotonic profile 

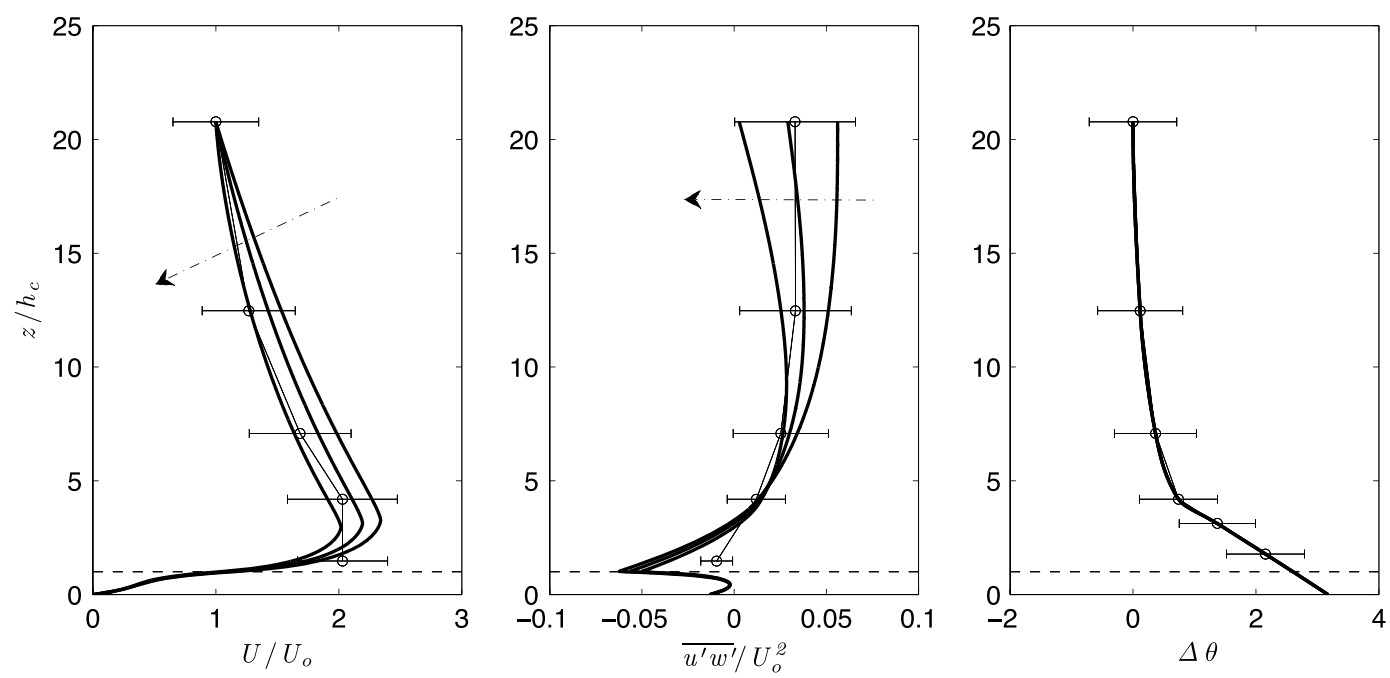

Figure 2. Comparison between measured (symbols with error bars) and modeled (lines) $U(z)$ and $\overline{u^{\prime} w^{\prime}}(z)$ averaged over $1 \mathrm{~h}$ for the night of 15 September. Horizontal error bars indicate one standard deviation derived from 5 min averages around the hourly mean. Dashed horizontal lines at $z / h_{c}=1$ show the canopy height. Arrows indicate direction of increasing $C_{p}(=0,5,10)$. For reference the measured $\Delta \theta(z)$ used to drive the model is shown.

for near-neutral conditions over flat terrain [e.g., Yi, 2008; Finnigan, 2000]. Near the ground, $U \approx 0$ so the drag force (scales as $\left.U^{2}\right)$ is small and $\partial / \partial z\left(\overline{u^{\prime} w^{\prime}}\right)$ must balance $\left(g / \theta_{o}\right) \sin (\alpha) \Delta \theta$ resulting in a negative deviation in $\overline{u^{\prime} w^{\prime}}$ from zero. Additionally, a nonzero $\partial P / \partial x$ amplifies this effect. The derivation here also makes clear that $C_{p}$ is only significant when larger-scale variations introduce large variability in wind direction. Figure 3 suggests that the optimal $C_{p}$ derived by minimizing the root-mean-squared error between measured and modeled $U(z)$ is largest for small $U_{o}$ and that small $U_{o}$ are, in fact (observed also by Davies and Thomson [1999], Turnipseed et al. [2003]), associated with the largest variance in wind direction (thereby sampling multiple slope angles). This trend is observed at all measurement heights (see Figure S1 in the supporting information), which implies that larger-scale pressure perturbations can penetrate down to the surface and that flow decoupling through the katabatic layer does not occur (unlike the case of a dense and tall forested canopy). For large $U_{o}$ or small variations in wind direction at $h_{j}$, the optimal $C_{p} \approx 0$ and this term can be ignored for a conventional katabatic jet along a homogeneous slope. However, if the wind direction fluctuations are large, presumably due to larger disturbances originating from the outer layer, then $C_{p} \approx 20$. Note that following the optimization of the $C_{p}$ value, the root-mean-squared error between measured and modeled $U(z)$ becomes independent of $U_{o}$ (i.e., the flow conditions above the katabatic jet).
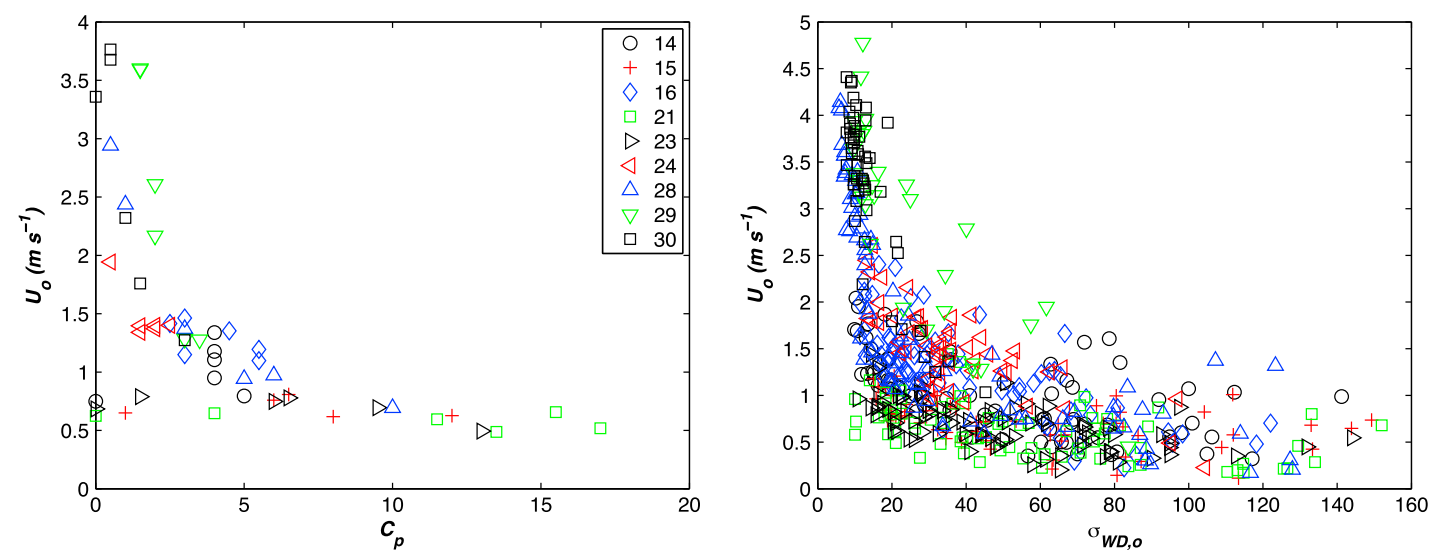

Figure 3. The variation of computed optimal $C_{p}$ as inferred by minimizing the root-mean-squared error (RMSE) between measured and modeled $U(z)$ for each $1 \mathrm{~h}$ run across nine nighttime runs in September (differentiated by symbols) for measured $U_{o}$. Note the increasing $C_{p}$ with decreasing $U_{o}$. Finally, the relation between the measured $U_{o}$ and $\sigma_{W D, o}$ is shown for all 5 min segments. 
Even in the cases when $C_{p} \approx 20$, this term contributes to no more than $20 \%$ of the mean momentum balance. Therefore, the outer pressure term can be significant, but as expected, the momentum budget is mainly a balance between buoyancy and frictional mechanisms. Naturally, $C_{p}$ may also encode other processes such as local advection and subsidence that need not be independent of $z$. However, lower values of $C_{p}$ are associated with low root-mean-squared error and higher values of $U_{o}$, when advective effects are more likely to have importance. Hence, advection likely plays a small role in the momentum budget here, and its omission is a reasonable assumption (see also supporting information). In contrast, Horst and Doran [1986] show results for a site where vegetation and topography are highly variable in space, and advection cannot be neglected a priori, likely because of these nonuniformities along the slope. Figure 3 makes clear that deviations between modeled and measured $U(z)$ can be partially corrected via a $C_{p}$ that is significantly linked to variability in wind direction. This link is further established by Figure $S 2$ in the supporting information that shows the correlations between $U$ and mean wind direction, which is characterized by a Gaussian distribution (Figure S3).

\section{Conclusions}

A one-dimensional mean momentum balance formulation is developed for describing the katabatic jet on steep slopes covered with vegetation. The main novelty is inclusion of the effects of the short vegetation and the weak larger-scale pressure perturbations that cause large fluctuations in wind direction. The model explicitly reveals how these two additions impact the location of the peak velocity within the katabatic jet. Because of its simplified structure, the approach proposed here can be used to complement classical wall functions used to bridge the land surface with atmospheric flows in large-scale numerical models of mountainous terrain. It can also aid in field experiment designs aimed at resolving the structure of turbulence within the katabatic jet. This latter topic is now receiving broad experimental attention as approaches to quantifying $\mathrm{CO}_{2}$ drainage at night are becoming a central research focus to correcting eddy covariance based flux measurements aimed at estimating carbon balances over complex topography.

\section{Acknowledgments}

The data for this work can be obtained via email from the corresponding author. This research was primarily funded by the Swiss National Science Foundation (SNSF-200021-134892) and the Competence Center for Environmental Sustainability (CCES-SwissEx) of the ETH domain. Katul acknowledges the National Science Foundation (NSF-EAR-1013339, NSF-AGS-1102227, and NSF-CBET-103347), the United States Department of Agriculture (2011-67003-30222), the U.S. Department of Energy (DOE) through the office of Biological and Environmental Research (BER)Terrestrial Ecosystem Science (TES) Program (DE-SC0006967), and the Binational Agricultural Research and Development (BARD) Fund (IS-4374-11C). Pardyjak acknowledges the Office of Naval Research Award N00014-11-1-0709. Special thanks to the Commune d'Orsiéres for logistical support for field campaigns in Val Ferret.

The Editor thanks two anonymous reviewers for assistance evaluating this manuscript.

\section{References}

Belcher, S. E., and N. Wood (1996), Form and wave drag due to stably stratified turbulent flow over low ridges, Q. J. R. Meteorol. Soc., 122(532), 863-902.

Brustaert, W. (1982), Evaporation Into the Atmosphere, Theory, History and Application, Kluwer Acad., Dordrecht, Netherlands.

Burns, S. P., J. Sun, D. H. Lenschow, S. P. Oncley, B. B. Stephens, C. Yi, D. E. Anderson, J. Hu, and R. K. Monson (2011), Atmospheric stability effects on wind fields and scalar mixing within and just above a subalpine forest in sloping terrain, Boundary Layer Meteorol., 138(2), 231-262.

Chen, H., and C. Yi (2012), Optimal control of katabatic flows within canopies, Q. J. R. Meteorol. Soc., 138(667), 1676-1680.

Davies, B. M., and D. J. Thomson (1999), Comparisons of some parametrizations of wind direction variability with observations, Atmos. Environ., 33(29), 4909-4917.

Duynkerke, P. G. (1999), Turbulence, radiation and fog in dutch stable boundary layers, Boundary Layer Meteorol., 90(3), $447-477$.

Finnigan, J. (2000), Turbulence in plant canopies, Annu. Rev. Fluid Mech., 32(1), 519-571.

Foken, T. (2008), Micrometeorology, Springer, Berlin, Germany.

Froelich, N. J., and H. P. Schmid (2006), Flow divergence and density flows above and below a deciduous forest: Part II. Below-canopy thermotopographic flows, Agric. For. Meteorol., 138(1), 29-43.

Froelich, N. J., H. P. Schmid, C. S. B. Grimmond, H.-B. Su, and A. J. Oliphant (2005), Flow divergence and density flows above and below a deciduous forest: Part I. Non-zero mean vertical wind above canopy, Agric. For. Meteorol., 133(1), 140-152.

Garrett, A. J. (1983), Drainage flow prediction with a one-dimensional model including canopy, soil and radiation parameterizations, J. Clim. Appl. Meteorol., 22(1), 79-91.

Haiden, T., and C. D. Whiteman (2005), Katabatic flow mechanisms on a low-angle slope, J. Appl. Meteorol., 44(1), $113-126$.

Horst, T., and J. Doran (1986), Nocturnal drainage flow on simple slopes, Boundary Layer Meteorol., 34(3), 263-286.

Jiménez, M. A., and J. Cuxart (2005), Large-eddy simulations of the stable boundary layer using the standard Kolmogorov theory: Range of applicability, Boundary Layer Meteorol., 115(2), 241-261.

Katul, G. G., L. Mahrt, D. Poggi, and C. Sanz (2004), One-and two-equation models for canopy turbulence, Boundary Layer Meteorol., 113(1), 81-109.

Laughlin, G. P., and J. D. Kalma (1987), Frost hazard assessment from local weather and terrain data, Agric. For. Meteorol., 40(1), 1-16.

Luo, Z., and Y. Li (2009), Influence of plant canopy on the katabatic winds, paper presented at Seventh International Conference on Urban Climate, Yokohama, Japan.

Luo, Z., Y. Li, and C. Yi (2009), Analytical solution of cold-air-drainage flow within and above forest canopy, paper presented at Second International Conference on Countermeasures to Urban Heat Islands, Berkeley, Calif.

Mahrt, L. (1982), Momentum balance of gravity flows, J. Atmos. Sci., 39, 2701-2711.

Mahrt, L. (1998), Stratified atmospheric boundary layers and breakdown of models, Theor. Comput. Fluid Dyn., 11(3), $263-279$.

Mahrt, L., D. Vickers, R. Nakamura, M. R. Soler, J. Sun, S. Burns, and D. H. Lenschow (2001), Shallow drainage flows, Boundary Layer Meteorol., 101(2), 243-260.

Manins, P. C., and B. L. Sawford (1979), A model of katabatic winds, J. Atmos. Sci., 36, 619-630.

Monti, P., H. J. S. Fernando, M. Princevac, W. C. Chan, T. A. Kowalewski, and E. R. Pardyjak (2002), Observations of flow and turbulence in the nocturnal boundary layer over a slope, J. Atmos. Sci., 59(17), 2513-2534. 
Nadeau, D. F., E. R. Pardyjak, C. W. Higgins, and M. B. Parlange (2012), Similarity scaling over a steep alpine slope, Boundary Layer Meteorol., 147(3), 401-419.

Nadeau, D. F., E. R. Pardyjak, C. W. Higgins, H. Huwald, and M. B. Parlange (2013), Flow during the evening transition over steep alpine slopes, Q. J. R. Meteorol. Soc., 139(672), 607-624.

Pahlow, M., M. B. Parlange, and F. Porté-Agel (2001), On Monin-Obukhov similarity in the stable atmospheric boundary layer, Boundary Layer Meteorol., 99(2), 225-248.

Pardyjak, E. R., H. J. S. Fernando, J. C. R. Hunt, A. A. Grachev, and J. Anderson (2009), A case study of the development of nocturnal slope flows in a wide open valley and associated air quality implications, Meteorol. Z., 18(1), 85-100.

Rao, K. S., and H. F. Snodgrass (1981), A nonstationary nocturnal drainage flow model, Boundary Layer Meteorol., 20(3), 309-320.

Raupach, M. R., and J. J. Finnigan (1997), The influence of topography on meteorlogical variables and surface-atmosphere interactions, J. Hydrol., 190(3), 182-213.

Renfrew, I. A. (2004), The dynamics of idealized katabatic flow over a moderate slope and ice shelf, Q. J. R. Meteorol. Soc., 130(598), 1023-1045.

Simoni, S., S. Padoan, D. F. Nadeau, M. Diebold, A. Porporato, G. Barrenetxea, F. Ingelrest, M. Vetterli, and M. B. Parlange (2011), Hydrologic response of an alpine watershed: Application of a meteorological wireless sensor network to understand streamflow generation, Water Resour. Res., 47(10), W10524, doi:10.1029/2011WR010730.

Sun, J., et al. (2007), $\mathrm{CO}_{2}$ transport over complex terrain, Agric. For. Meteorol., 145(1-2), 1-21.

Turnipseed, A. A., D. E. Anderson, P. D. Blanken, W. M. Baugh, and R. K. Monson (2003), Airflows and turbulent flux measurements in mountainous terrain: Part 1. Canopy and local effects, Agric. For. Meteorol., 119(1), 1-21.

Whiteman, C. D. (2000), Mountain Meteorology: Fundamentals and Applications, Oxford Univ. Press, New York.

Whiteman, C. D., S. Zhong, W. J. Shaw, J. M. Hubbe, X. Bian, and J. Mittelstadt (2001), Cold pools in the Columbia basin, Weather Forecast, 16(4), 432-447.

Wilczak, J. M., S. P. Oncley, and S. A. Stage (2001), Sonic anemometer tilt correction algorithms, Boundary Layer Meteorol, 99(1), 127-150. Yamada, T. (1983), Simulations of nocturnal drainage flows by a $\mathrm{q}^{2}$ I turbulence closure model, J. Atmos. Sci., 40(1), 91-106. Yi, C. (2008), Momentum transfer within canopies, J. Appl. Meteorol. Clim., 47(1), 262-275.

Yi, C., R. K. Monson, Z. Zhai, D. E. Anderson, B. Lamb, G. Allwine, A. A. Turnipseed, and S. P. Burns (2005), Modeling and measuring the nocturnal drainage flow in a high-elevation, subalpine forest with complex terrain, J. Geophys. Res., 110, D22303, doi:10.1029/2005JD006282. 